\title{
Comparision of Ankle Joint Range of Motion on Balance Score in Healthy Young and Adult Individuals
}

\author{
Gaur $^{1}$, K. Davinder, Ashutosh ${ }^{2}$
}

${ }^{1}$ Asst. Professor, Banarsidas Chandiwala Institute of Physiotherapy (BCIP), New Delhi, Delhi NCR, India

${ }^{2}$ Physiotherapist, Banarsidas Chandiwala Institute of physiotherapy (BCIP), New Delhi, Delhi NCR, India

\begin{abstract}
Background and Introduction: Flexibility at the ankle joints provides an important contribution to safe execution of many functional tasks in our day to day activities like walking, negotiating stairs, rising from a chair and added efficiency in the maintenance of postural stability. Lack of mobility at ankle joint leads to inefficient balance and frequent falls. A number of studies have been performed for falls in elderly and analyzed the composition of sway in adults and "healthy" elderly people. In this study comparison of ankle joint range of motion on balance score in healthy young and adult individuals is done. Method: 30 subjects were selected according to the inclusion and exclusion criteria. Subjects were measured for active and passive ankle dorsiflexion and plantar flexion in high sitting position to measure range of motion at ankle joint. Then subjects went through Functional reach test and Timed up and go test and score was measured and noted. Results: Analysis of the relation between balance score and joint range of motion showed that ankle joint range of motion and balance are mutually dependent. Conclusion: The result of study shows that there is decrease in ankle joint range of motion with increasing age and thus affects balance also.

Key Words: Ankle joint range of motion, balance score
\end{abstract}

\section{Introduction}

The ankle is a complex joint that connects the foot to the lower leg. A hinge joint formed by the articulation of the tibia and the fibula with the talus below also called mortise joint. It bears up to eight times the body weight when one runs. Normal ankle function is needed to walk with a smooth and nearly effortless gait. The muscles, tendons, and ligaments that support the ankle joint work together to propel the body (Chaurasia, 2012). Flexibility at the ankle joints provides an important contribution to safe execution of many functional tasks (e.g. walking, negotiating stairs, rising from a chair) and added efficiency in maintenance of postural stability (Nitz \& Nancy, 2004). Consequently it is impossible to stand motionless as even when standing quietly on both feet, the body sways over its base of support. The basic requirement for standing balance is that the position of the body's centre of mass is held within the boundaries of the base of support established by the feet (Maki \& McIlroy, 1998). Flexibility at the ankle joint is directly related to the balance. Balance is defined as the ability to control body mass or centre of gravity to base of support (Gordon \& Ghez, 1991). Balance is a complex process involving reception and integration of sensory inputs and the planning and execution of movements to achieve a goal requiring upright posture. The ability to control postural balance is a prerequisite to performing many of the 
activities of daily living, and underpins the ability to maintain an independent lifestyle. Balance control is a complex system of coordinated muscular responses, dependent on somatosensory, vestibular and visual information (Johansson \& Magnusson, 1991). Inaccurate or insufficient information from any of these sensory receptors or impairment affecting the processing of these messages can disturb the triggering and regulation of movement (Quoniam et $a l, 1995)$. The ability to maintain balance in stance and locomotion is thus dependent on the integrity of the receptor systems. Ankle movements are also necessary for muscular responses used to maintain perturbations to balance, such as rapid compensatory stepping movements. Recent studies highlight the importance of compensatory stepping to preserve stability, and the spatial and temporal demands placed on the control of this reaction. Age-related changes in the control of stepping could greatly influence the risk of falling (Mcllroy \& Maki, 1996).

Loss of joint range is considered to be a part of normal aging process. Therefore, decreased ankle range of motion with age may require altered movement patterns which may compromise balance, thus limiting functional activities such as ambulation with aging; there is a significant deterioration in balance control mechanisms (Woollacott, \& ShumwayCook, 1990). Loss of balance and falls in the elderly constitute a major problem associated with human suffering as well as high costs for society (CDC, 2006) Falls might occur during various daily activities, such as tripping or tangling the feet, reaching movements or bending (Maki \& McIlroy, 1996). Many of these activities are constrained by limits of stability (LOS). The cause of falls in the elderly is multifactorial (Tinetti, 2003). Amongst the many factors described, the fact that the aging process results in reduced joint flexibility and reduced afferent sensory information is well established. All joints show a significant reduction in range of motion (ROM) with age. Ankle dorsiflexion (knee extended) shows the greatest age-related decline (James \& Parker, 1989). Decrease in dorsiflexion ROM is associated with normal aging in both men and women (Lung et al, 1996, Gadjosik et al, 1999). Fallers show a reduced ankle ROM (Kemoun et al, 2002). LOS can be described as the maximum distance a person can intentionally displace his/her centre of gravity, and lean his/her body in a given direction without losing balance, stepping or grasping. Accordingly, one's LOS capacity is likely to be an important prerequisite for the successful planning and execution of movements such as using a step stool to reach into a high cabinet as well as bending over from standing position to pick up an object from the floor. In the human body the high centre of gravity, together with the small base of support in standing, place the body in unstable equilibrium. Balance is continually challenged by destabilizing internal perturbations from neuromuscular noise and hemodynamic (Conforto et al, 2001), as well as by the force of gravity, perturbations from volitional movement (e.g. turning, bending) and interactions with the environment. Adequate postural control depends on the spatial and temporal integration of vestibular, visual, 
and somatosensory information about the motion of the head and body, and the generation of appropriate responses to that motion. The increased incidence of falls in the older population suggests that one or more of these components degenerate with age. Diminished visual, vestibular, and somatosensory function and slowing of sensorimotor processing all occur with normal aging, and older people are also at higher risk for many diseases affecting the peripheral and central nervous system (Horak et al, 1989). In addition to decreases in muscle strength and slower neural processing, there are a number of sensory changes that may contribute to unsteadiness in the elderly. These include age-related decreases in the number of hair cells in both the canals and the otolith organs, and in the number of nerve fibers in the vestibular nerve, eventually resulting in reduced vestibular excitability (Rosenhall, 1973, Bergstrom, 1973, Rosenhall \& Rubin, 1975). Elderly also show a significant decrease in the sensitivity of vision to low frequency spatial motion (Sekuler \& Hutman, 1980). The study was conducted to compare the ankle joint range of motion on balance score of healthy young and adult individuals

\section{Materials and Methods:}

The study was conducted on 30 subjects belonging to Delhi region with random sample selection. Individuals who fulfilled the inclusion and exclusion criteria and gave written consent for participation were selected and divided into two groups made for the purpose of comparison including randomly allocated 15 subjects in each group. The purpose of this study was explained to the subjects. All the subjects fulfilling the inclusion and exclusion criteria who volunteered to participate in the study were described verbally about the procedure to be used in the study. Testing was performed only after informed consent was taken from the subjects.

Following procedures were performed Measurement of dorsiflexion and plantar flexion range-

Subject was placed in high sitting position on a plinth. A double arm goniometer was used to measure the range of motion. Fulcrum was placed on the lateral malleoli, stable arm of goniometer along the tibia in such a manner that a imaginary line joining the lateral malleoli and head of fibula. Foot was maintained in 90 degree position to the tibia, movable arm of the gonio was placed parallel to $\mathrm{V}$ Metatarsal. Now subject was asked to move the ankle upward (active dorsiflexion). Then after maintaining the initial position, subject was asked to take his foot in downward direction (active plantar flexion). After that, whole procedure was repeated passively to take passive dorsi flexion and planter flexion range of motion.

Functional Reach Test (FRT) (Duncan, 1991)

- Subject staood with feet shoulder width apart and with the arm raised to 90 degrees of flexion along a yardstick placed at humeral level.

- Without moving the feet, the subject was asked to reach as far forward as possible while maintaining balance.

- 3 trials were given.

- The distance reached was measured, average of the three trials was taken and compared to age-related norms. While measurement were taken, it was kept in mind that readings were taken at 
metacarpo phalangeal joint line up and fist closed. Less than 7 inch reach is associated with frailty and increased fall risk.

Timed Up \& Go Test (TUG) (Podsiadlo, and Richardson, 1991)

Instructions:

The person was allowed to wear their usual footwear and allowed to use any assistive device they normally use.

- The subject was asked to sit in the chair with their back to the chair and their arms resting on the arm rests.

- The subject was asked to stand up from a standard chair and walk a distance of $10 \mathrm{ft} .(3 \mathrm{~m})$ and then turn around, walk back to the chair and sit down again. The time was measured from the point the subject started to rise from the chair to the end when he or she returned to the chair and sat down. Three trials were given and the average time of the three attempts was calculated. Data was collected and for the purpose of comparison and were analyzed to obtain the result.

\section{Results \& Discussion}

Table 1: Physical characteristics of the subjects

\begin{tabular}{|c|c|c|c|}
\hline \multicolumn{3}{|c|}{ GROUP A } & GROUP B \\
\hline \multicolumn{2}{|l|}{$\mathbf{N}$} & 15 & 15 \\
\hline \multicolumn{2}{|c|}{ Male (N) } & 6 & 8 \\
\hline \multicolumn{2}{|c|}{ Female $(\mathbf{N})$} & 9 & 7 \\
\hline \multicolumn{2}{|c|}{ Mean age (SD) } & $21.53(0.915)$ & $51.46(5.208)$ \\
\hline $\begin{array}{l}\text { Mean } \\
\text { (SD) }\end{array}$ & height & $5.25(0.393625)$ & $5.18(0.447413)$ \\
\hline $\begin{array}{l}\text { Mean } \\
\text { (SD) }\end{array}$ & weight & $\begin{array}{c}58.73 \\
(13.46671)\end{array}$ & $\begin{array}{c}61.46 \\
(11.54412)\end{array}$ \\
\hline
\end{tabular}

Table 2: Comparison of mean active dorsiflexion and planter flexion range of motion in the two groups

\begin{tabular}{lcc}
\hline & $\begin{array}{c}\text { Mean Active } \\
\text { Dorsiflexion ROM } \\
\text { (SD) }\end{array}$ & $\begin{array}{c}\text { Mean Active } \\
\text { Plantar flexion } \\
\text { ROM (SD) }\end{array}$ \\
\hline GROUP A & $\mathbf{1 5 . 3 3 ( 2 . 4 3 9 7 5 )}$ & $43.66(6.904105)$ \\
GROUP B & $\mathbf{1 4 . 1 3 ( 2 . 0 3 0 7 1 7 )}$ & $41.26(3.261609)$ \\
\hline
\end{tabular}

Table 3: Comparison of Mean passive dorsiflexion and plantar flexion range of motion in in the two groups

Mean Passive Mean Passive

Dorsiflexion ROM, Planter flexion

(SD) ROM, (SD)

\begin{tabular}{lll}
\hline GROUP A & $24.6(3.5817)$ & $60.4(7.962412)$ \\
GROUP B & $20.2(3.820995)$ & $50.4(7.509993)$ \\
\hline
\end{tabular}

Table 4: Comparison of Mean Functional Reach score (FRT) Scores among the two groups

\begin{tabular}{lcc}
\hline & GROUP A & GROUP B \\
\hline Mean FRT score & 8.05 & 4.19 \\
SD & 1.49 & 0.70 \\
\hline
\end{tabular}

Table 5: Comparison of Mean Timed Up \& Go score (TUG) Scores among the two groups

\begin{tabular}{lcc}
\multicolumn{3}{c}{ (TUG) Scores among the two groups } \\
\hline Mean TUG score & GROUP A & GROUP B \\
SD & 0.787 & 11.155 \\
\hline
\end{tabular}

The results of present study showed a statistically significant lower ankle joint range of motion in the older age group. A number of studies have already revealed that this reduction in joint range is due to ageing. Ageing is a normal process in every individual's life and it can't be stopped. The process of ageing starts just after child takes birth. Ageing affects all the body parts ranging from cellular level to organ level. Some changes related to ageing have been observed in our joints also. Common changes related to increasing age seen in the joints areincreased stiffness of periarticular connective tissue, decreased movement about the ankle joint produced by a lower muscle force, loss of proprioceptors, decreased activity level.

Balance while walking, negotiating stairs, and even while standing need good flexibility, proprioception, and muscle strength in lower extremity. Ankle joint 
plays a very critical role while walking and standing. All joints show a significant reduction in range of motion (ROM) with age. Ankle dorsiflexion (knee extended) shows the greatest age-related decline (Tinetti, 2003). Several studies have revealed a decrease in dorsiflexion ROM with normal aging in both men and women (Lung et al, 1996, Gadjosik et al, 1999). In our study, a significant reduction in dorsiflexion and as well as in planter flexion range of motion with increasing age has been observed that is in accordance with the above reports. The reduction in movement is not only limited to active movement of ankle but also to the passive movement as well. Mean Active Dorsiflexion ROM of group A is found to be 15.33 while for group B it is 14.33. Mean Active Planter flexion ROM of group A is found to be 43.66 and that of group B is 41.26. Differences are also seen in passive movements of ankle also. mean passive dorsiflexion ROM of group A is 24.6 while for group B it is 20.2. mean passive plantar flexion ROM of group A is 60.4 and for group B it is 50.4, suggesting that with increasing age, periarticular structures of ankle lose their flexibility.

According to Kemoun et al, (2002), fallers show a reduced ankle ROM. Despite the atrophy of the ankle musculature which occurs with aging, passive resistive torque of stretched connective tissue shows an increasing trend in older subjects (Lung et al, 1996). Adequate ROM of the ankle and MTP joints is an important prerequisite to enable balance and locomotion which are essential daily activities (Lung et al, 1996). and have found an age-related decrease in flexion of the first metatarso phalangeal (MTP) joint. In addition to the musculoskeletal aspects, control of balance requires coordinated activity of the neuromuscular system. Accurate sensory inputs are necessary to organize motor programs and to generate effective motor output responses (Lung et al, 1996). A good amount of proprioceptors and coordination is a prerequisite of good balance. However decrease in proprioceptors is also taken as a part of normal ageing process. Different parameters are used to measure the balance score. Out of the different parameters in the present investigation functional reach test and timed up and go test as a measure of balance score was taken. Significant differences are also found after comparing the balance score of group A and group B. Mean FRT score for group $\mathrm{A}$ is found to be 8.05 while for group B that comprise of older subjects, it is 4.19. So, from the above mentioned scores it is clear that group A individuals who are younger demonstrate significantly greater balance scores than group B individuals who are older. Scores predict that chances of falls in group B individuals are quiet higher than group A individuals. TUG scores also show that mean TUG score for group A is 6.78 and for group B it is 11.15 . It shows that to cover particular distance group B individuals took much more time than group A individuals. It is suggestive of lowering of gait with age and lack of balance \& flexibility

Interpretation- From the above mentioned data, it is clear that there is significant differences in the mean FRT values of group A \& group B. Group A individual are having higher mean FRT values than group B individuals, showing good 
flexibility of ankle joint and have good balance.

From the above mentioned data, it is also clear that there is significant difference in the mean TUG values of group A \& group B. According to the scale mentioned by Hamilton County, individuals of group $\mathrm{A}$ are mostly independent $(<20)$ while individuals of group B are Freely Mobile $(<10)$, group B individuals are having comparatively higher risk of falls than group A individuals.

\section{References}

Chaurasia, B. D. 2012. Human Anatomy Lower Limb, regional and applied dissection and clinical lower limb abdomen and pelvis (volume - 2) 5 edition (regional and applied dissection and clinical lower limb abdomen and pelvis. Vol-2, Edition-6. CBS Publishers, Daryaganj, Daryaganj, New Delhi, Delhi,

Nitz, Jennifer, C., \& Choy, Nancy, Low. 2004. The relationship between ankle dorsiflexion range, falls and activity level in women aged 40 to 80 years. New Zealand Journal of Physiotherapy., 32(3): 121-125.

Maki, B.E., McIlroy, W.E., 1998. Control of compensatory stepping Reactions: age related impairment and the potential for remedial Intervention. Physiotherapy. Theory. Prac., 15: 69-90.

Gordon, J., Ghez C. 1991. Muscle receptors, www.linkinghub.elsevier.com/retrieve/pii/S096706 6104002266.

Johansson, R., Magnusson, M. 1991. Human postural dynamics. Crit. Rev. Biomed. Eng., 18: 413-37.

Quoniam, C., Hay, L., Roll, J-P., Harley, F. 1995. Age effects on reflex and postural responses to propriomuscular inputs generated by tendon vibration, J. Gerentol Bio. Sci., 50A: B155-65.

William, E. McIlroy \& Brian E. Maki 1996. Age related changes in compensatory stepping in response to unpredictable perturbations. J. Gerontology: Medical Sciences, 51A (6): M289 -M296.

Woollacott, M..H.., Shumway-Cook, A., 1990. Changes in posture control across the life span: a systems approach. Phys. Ther., 70: 799-807.
Centers for Disease Control and Prevention (CDC) 2006. Fatalities and injuries from falls among older adults - United States, 1993-2003 and 2001-2005. MMWR Morb Mortal Wkly Rep 2006; 55: 1221-4.

Maki, B.E., McIlroy, W.E. 1996. Postural control in the older adult. Clin. Geriatric Med., 12: 635-58.

Tinetti, M.E. 2003. Preventing falls in elderly persons. New England Journal of Medicine 348: 42-9.

James, B, Parker A.W. 1989. Active and passive mobility of lower limb joints in elderly men and women. American Journal of Physical Medicine, 68(4): 162-7.

Gadjosik, R.L., Vander Linden, DW, Williams, AK. 1999. Influence of age on length and passive elastic stiffness characteristics of the calf muscle-tendon unit of women. Physical Therapy, 79: 827-38.

Lung, M.W., Hartsell, H.D., Vandervoort, A.A. 1996. Effects of aging on joint stiffness: Implications for exercise. Physiotherapy Canada, 48(2): 96-106.

Kemoun, G., Thoumie, P., Boisson, D., Guieu, J.D. 2002. Ankle dorsiflexion delay can predict falls in elderly. Journal of Rehabilitation Medicine, 34: 278-83.

DeLuca, C.J., LeFever, R.S., McCue, M.P., Xenakis, A.P., 1982. Control scheme governing concurrently active human motor units during voluntary contractions. J. Physiol. (London), 329: 129-142.

Conforto, S., Schmid, M., Camomilla, V., D_Alessio, T., Cappozzo, A., 2001. Hemodynamic as a possible internal mechanic disturbance to balance. Gait Posture, 14: 28-35.

Horak, F.B., Shupert, C.L. Mirka, A. 1989. Components of postural decontrol in the elderly: a review. Neurobiol. Aging, 10: 727-38.

Rosenhall, U. 1973. Degenerative patterns in the aging human vestibular neuro-epithelia. Acta Otolaryngologica, 76: 208-20.

Bergstrom, B. 1973. Morphology of the vestibular nerve. 3. Analysis of the calibres of the myelinated vestibular nerve fibers in man at various ages. Acta Otolaryngologica, 76: 331-8.

Rosenhall, U., Rubin, W. 1975. Degenerative changes in the human vestibular sensory epithelia. Acta Otolaryngologica, 79: 67-80.

Sekuler, R., Hutman, L.P. 1980. Spatial vision and aging. I: Contrast sensitivity. J. Gerontology, 35: 692-9.

Pamela, W., Duncan, Stephanie Studenski, Julie Chandler and Barbara Prescott. 1992.: Functional Reach: Predictive Validity in a Sample of Elderly Male Veterans, J. Gerontol., 47(3): M93-M98..

Diane Podsiadlo, and Sandra Richardson. 1991. Timed Get-Up and Go Test, J. Am.Geriatric Soc., 39: 142-148. 